\title{
Criminologie
}

\section{Comment règle-t-on le problème du vol à l'étalage dans les espaces commerciaux français?}

\section{Frédéric Ocqueteau et Marie-Lys Pottier}

Volume 31, numéro 2, automne 1998

La sécurité privée

URI : https://id.erudit.org/iderudit/017419ar

DOI : https://doi.org/10.7202/017419ar

Aller au sommaire du numéro

Éditeur(s)

Les Presses de l'Université de Montréal

ISSN

0316-0041 (imprimé)

1492-1367 (numérique)

Découvrir la revue

Citer cet article

Ocqueteau, F. \& Pottier, M.-L. (1998). Comment règle-t-on le problème du vol à l'étalage dans les espaces commerciaux français ? Criminologie, 31(2), 69-85.

https://doi.org/10.7202/017419ar
Résumé de l'article

Based on the quantitative data derived from the files of various large shopping centers in France, a set of criminal events (i.e. shop-lefting) are analysed. The article meets two concerns : (1) assessing the autonomy of private police via a calculation of the proportion of non-referals of cases to the police and to court and (2) understanding the signification given by private police agents to practices of externalisation of disputes. The key determinant relativising the concept of private policing is the confidence negotiated by the directors of large distribution organizations with public police. In fact, private regulation is not necessarily contradictory with legality, if public actors define their rules on private spaces receiving the public, in matter of policing, after having taken the exact measure of the incidents which affect them. 
COMMENT RÈGLE-T-ON LE PROBLÈME DU VOL À L'ÉTALAGE DANS LES ESPACES COMMERCIAUX FRANÇAIS ?

Based on the quantitative data derived from the files of various large shopping centers in France, a set of criminal events (i.e. shoplefting) are analysed. The article meets two concerns : (1) assessing the autonomy of private police via a calculation of the proportion of non-referals of cases to the police and to court and (2) understanding the signification given by private police agents to practices of externalisation of disputes. The key determinant relativising the concept of private policing is the confidence negotiated by the directors of large distribution organizations with public police. In fact, private regulation is not necessarily contradictory with legality, if public actors define their rules on private spaces receiving the public, in matter of policing, after having taken the exact measure of the incidents which affect them.

\section{INTRODUCTION}

Une hypothèse très féconde pour expliquer la croissance des ressources en matériels et agents de sécurité privée provient d'une théorisation de la propriété privée de masse (Shearing et Stenning, 1983, 1987) dérivée du constat de l'expansion historique des domaines privés ouverts à des publics nombreux.

Cette expansion de la patrimonialité des espaces récréatifs ou commerciaux aurait provoqué chez leurs usagers des manières de les habiter conduisant à des changements fondamentaux dans les modalités de la gestion de l'ordre (policing). N'étant plus en présence d'espaces publics (la rue, les routes) quadrillés par les forces de polices publiques, ni de domaines strictement fermés du propriétaire exerçant sa surveillance selon ses propres moyens, surveillance et protection privée fonctionneraient selon des modalités

1. Chargé de recherche au Groupe d'analyse des politiques publiques (UPR - CNRS), Bâtiment Cournot, École normale supérieure, 61, avenue du Président Wilson, 94235, CachanCedex, France.

2. Ingénieur d'études au CESDIP / CNRS, Immeuble Edison, 43, boul. Vauban, 78280 , Guyancourt, France. 
différentes du passé : la prévention des pertes (loss prevention) liée aux conséquences d'accidents ou actes malveillants l'emporterait sur la nécessité d'arrêter et de punir les délinquants fautifs. Autrement dit, dans ces espaces, le calcul instrumental du coût de l'exclusion des perturbateurs indisciplinés étant mis en balance avec les bénéfices escomptés de leur mise au pas disciplinaire, l'ordre lié au bon fonctionnement de la vie des organisations primerait sur la philosophie du respect de la loi et de l'ordre (law and order) recherchée dans l'espace public. Ces deux données inédites (changement dans la nature des espaces de vie et de la nature de la gestion de l'ordre) font aujourd'hui presque figure de paradigme dans l'orbite de la criminologie critique du contrôle social. Doublées par un mouvement de crise fiscale affectant les ressources publiques et une demande croissante de sécurité émanant de la société civile, les pouvoirs publics devraient, pour procurer la sécurité à un niveau satisfaisant, réapprendre la gouvernance locale de la prévention du crime en partenariat avec de nouveaux acteurs privés et les différentes communautés locales concernées (Crawford, 1997).

Lorsque l'on soumet à une vérification empirique ces grandes évolutions à partir d'enquêtes socio-criminologiques sur la gestion de l'ordre produit dans l'espace commercial (les grands centres d'achat sont, de ce point de vue, un terrain de prédilection, voir Davis, Lundman et Martinez, 1991 ; Farrington, Bowen, Buckle, Burns-Howell, Burrows et Speed, 1993 ; Hibbert et Shapland, 1993 ; Beck et Willis, 1995) le panorama dressé est moins ambitieux, la réalité sociale semblant par ailleurs plus complexe et ouverte. Notre propos ne visera pas à invalider le paradigme shearingien (il faudrait pour cela en produire une mouture alternative), mais cherchera plutôt à prêter attention aux nuances et aux contradictions de la réalité concrète auxquelles nous sommes plus sensibles. D'abord, parce que nous nous situerons à un niveau meso, sur une évaluation à moyen terme, et dans un contexte national différent. Ensuite, pour nous prémunir d'un alarmisme très fin de siècle sur le prétendu déclin du policing public (Bayley et Shearing, 1996).

Nous nous proposons de documenter le probième de la gestion de l'ordre commercial à la lumière d'une enquête quantitative nationale conduite auprès de dirigeants de grandes surfaces (Ocqueteau et Pottier, 1995b) ${ }^{3}$. Cette enquête fut complétée par des observations de terrain dans plusieurs centres commerciaux de la région parisienne particulièrement confrontés à des phénomènes d'insécurité de nature criminelle (Ocqueteau, 1993 ; Ocqueteau et

3. Un questionnaire a été administré par voie postale, en 1994, à l'ensemble des 954 directeurs d'unités de vente supérieures à $2500 \mathrm{~m}^{2}$ sur tout le territoire, le but étant d'atteindre des structures en taille suffisamment importantes susceptibles d'être dotées d'un service de vigilance, quelle qu'en soit la forme. Quatre cent onze réponses ( $43 \%$ ) furent largement suffisantes pour valider la représentativité de la population sous de multiples paramètres. 
Pottier, 1995a). Elle a fait ressortir à quel point la subjectivité vécue et conçue des gestionnaires à l'égard de diverses menaces assaillant leurs sites conditionnait aussi les usages de la protection. C'est néanmoins à l'occasion d'un examen approfondi de la gestion du contentieux de masse que constitue le vol à l'étalage (le risque du métier suprême) que l'on peut faire dériver toutes les représentations de la maîtrise des autres risques. C'est une extraordinaire occasion de montrer comment fonctionnent les liens d'interdépendances entre les modalités d'auto-protection des commerçants et les prises en charge des problèmes des commerçants par les autorités policières, judiciaires et autres.

La gestion du vol à l'étalage en tant que risque du métier, contentieux de masse et source d'insécurité parmi d'autres, les pratiques d'interpellation des auteurs et les négociations autour du (non) renvoi (Zauberman, 1982), sont en effet un merveilleux fil conducteur ou prétexte pour tester quantitativement et qualitativement la solidité de l'hypothèse d'une gestion privatisée du contrôle. Cela veut dire qu'en nous plaçant du côté de l'univers professionnel de ceux qui ont à concilier impératifs du commerce et paix commerciale dans leur domaine, face à la multiplicité des troubles de jouissance qui y surviennent, nous renversons la perspective pénalocentriste traditionnelle de la sociologie judiciaire des filières de contentieux. En effet, nous centrons essentiellement notre attention sur les modalités de la régulation interne des désordres dans les espaces commerciaux. Même si l'on conclut que les pratiques de protection et de sécurité des managers commerciaux ne sont jamais complètement dénuées d'arrière-pensées visant à instrumenter les autorités publiques à la politique conduite au sein de leur organisation, cette attitude méthodologique suggère une rupture radicale avec un courant d'analyse qui a encore trop souvent tendance à déduire les stratégies d'alimentation du système pénal de la configuration de ses produits (par ex., Kerckvoorde, 1978 ; Simmat-Durant, 1994 ; Aubusson, 1997), laissant dans l'ombre des pans entiers de régulations dont il n'a jamais connaissance.

La gestion du vol à l'étalage sera donc au cœur de nos développements successifs : en tant que délit publiquement sanctionnable et principal risque du métier dans le monde de la distribution de masse, nous en éclairerons les modalités de gestion quantitatives, puis nous expliquerons les variations des renvois de leurs auteurs par des attitudes plus ou moins bien prédisposées à l'égard des autorités policières et judiciaires, et nous montrerons enfin à quel point le degré différentiel de confiance en l'efficacité du travail des autorités conditionne les pratiques et les formes de l'auto-protection. Mais auparavant, nous procéderons à une nouvelle explication de la baisse constatée de la statistique publique disponible à propos des délits de vol à l'étalage. 


\section{CE QUE L'ON CONNAÎT DU CONTENTIEUX PUBLIC DU VOL À L'ÉTALAGE}

En France, une synthèse récente dédiée aux comptes du crime (Robert, Aubusson de Cavarlay, Pottier et Tournier, 1994 : 19 et suivantes) est restée mutique à propos d'un très éloquent infléchissement de la courbe statistique des faits constatés de vols à l'étalage à partir de l'année 1986. Une note annexée signalait seulement que jusqu'en 1987 la rubrique en question était libellée vols dans les magasins, ce qui laissait supposer une définition plus restrictive que vol à l'étalage. En réalité, aucune explication convaincante n'était apportée à ce phénomène brutal de baisse après la hausse spectaculaire de la décennie précédente.

\section{Tableau 1}

Vols à l'étalage constatés par la police et la gendarmerie

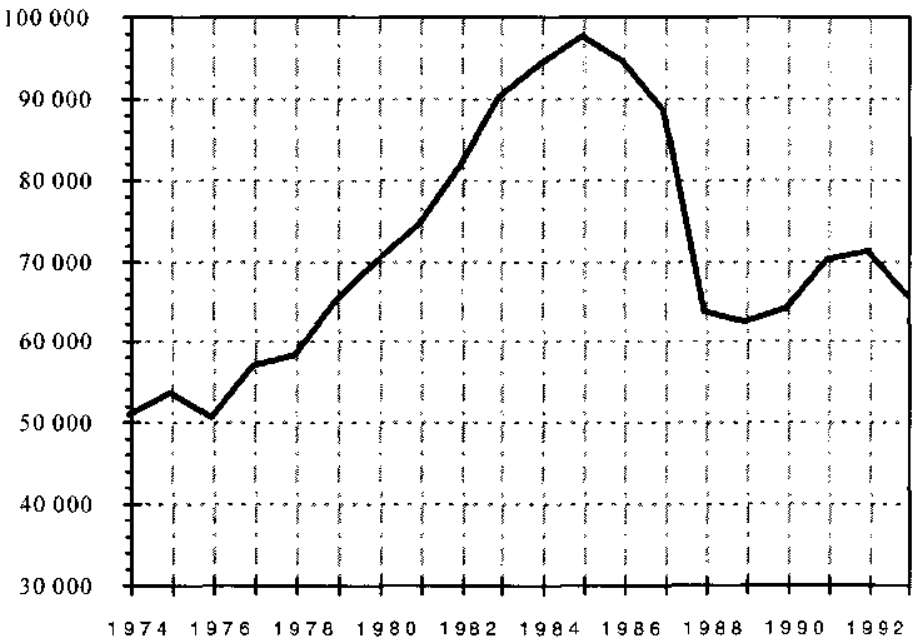

Source : Données actualisées dans Robert et al., 1994 (Annexe A2g)

À la même époque, des criminologues britanniques (Farrington et al., 1993 ; Farrington et Burrows, 1993) apportaient des précisions d'importance décisive dans l'explication d'un phénomène analogue de diminution statistique (d'un tiers) des vols à l'étalage constaté par les services du Home Office entre 1985 et 1989. Ayant enquêté auprès de 16 chaînes de détaillants pour comprendre les raisons de cette baisse, ces auteurs écartaient comme non plausibles un certain nombre de paramètres. Leur enquête montrait que le nombre des détectives de magasins n'ayant pas subi de variations 
significatives durant le laps de temps étudié (l'hypothèse étant que plus on mobilise ces agents, plus ils ont de chances théoriques de détecter de voleurs) et le nombre des interpellés étant lui-même resté à peu près constant, la seule explication satisfaisante de la chute était que les déclarations de vols à la police avaient significativement diminué. Ils en imputaient la raison à un recours de plus en plus fréquent à des procédures informelles de traitement des affaires en dehors des circuits publics.

Il y a douze ans, par une mesure passée complètement inaperçue en France, la Chancellerie a tenté de porter remède au contentieux de masse constitué par le vol à l'étalage en en privatisant partiellement la gestion sous le contrôle des Procureurs. Une circulaire du 10 juillet 1985 (Crim, 85-12E) instituait, à titre facultatif, la procédure dite de dépôt de plainte simplifiée (DPS). Elle laissait aux Procureurs le soin de définir un seuil minimal de préjudice en dessous duquel l'auteur interpellé en flagrant délit par le service de vigilance du commerçant pouvait échapper à une poursuite judiciaire sous certaines conditions : accepter de décliner son identité, admettre sa culpabilité et le montant du vol, restituer ou indemniser les objets volés, et signer un formulaire diligenté au Parquet, lequel décidait, le cas échéant, de classer l'affaire après admonestation de l'auteur, dûment averti des risques de poursuite et de sanction encourus en cas de récidive.

À la lumière de l'utilisation de cette procédure et à l'instar des chercheurs anglais, nous imputons l'explication de la baisse enregistrée dès 1986 à la mise en place de la nouvelle procédure. En 1988, il est hautement probable que la chute spectaculaire (de l'ordre de $25 \%$ ) ait correspondu à un effet d'anticipation policière harmonisé avec la nouvelle procédure de dépôt de plainte simplifiée : la police aurait ainsi appris très vite à ne plus enregistrer en main courante les vols dont elle a connaissance, inférieurs aux seuils de préjudice décidés par les services du Procureur dans chaque ressort judiciaire. Pour confidentielle que soit restée cette procédure, elle a produit et continue de produire des effets statistiques non négligeables sur le contentieux du vol à l'étalage. Désormais, les contentieux du vol à l'étalage ne pourront plus être analysés qu'à leur source réelle de régulation : à l'aune des stratégies de régulation des victimes corporatives. C'est dire le besoin de systématiser l'évaluation de la productivité de leur service de protection au moins dans ce domaine. La présente enquête avait pour but de s'y essayer à grande échelle, de quantifier et d'expliquer les renvois ou non-renvois des voleurs interpellés par les services de vigilance à la police et à la justice, afin de comprendre les mécanismes d'anticipation de ces agences, plutôt qu'à partir des caractéristiques biographiques du voleur, question traditionnellement plutôt bien documentée par la criminologie critique (Faugeron, Fichelet et Robert, 1977 ; Feuerverger et Shearing, 1982) ; et de rendre compte, autour 
de ces questions, des rationalités à l'œuvre dans les pratiques des victimes travaillant en relation plus ou moins lâche avec les autorités publiques.

\section{DONNÉES INÉDITES SUR L'EFFECTIVITÉ DE LA POLICE PRIVÉE EN MATIÈRE DE VOLS À L'ÉTALAGE}

Les données fournies concernent les années 1989 à 1993. Elles portent sur le nombre des interpellations annuelles effectuées par les agents d'inspection, le nombre de cas où police et gendarmerie ont été alertées, et le nombre de cas où l'on a recouru à la procédure de DPS devant le Procureur. Pour chaque établissement, une mesure des taux d'alerte et d'enclenchement d'une procédure formelle a été calculée par rapport au nombre des interpellations effectives, les deux actions étant indépendantes l'une de l'autre. De façon à montrer une évolution quantifiée sur cinq ans des interpellations et procédures consécutives, une valeur moyenne pour chaque variable créée a été calculée, compte tenu de la taille des établissements ventilés en gros $\left(>10000 \mathrm{~m}^{2}=15 \%\right)$, moyens (entre 5000 et $10000 \mathrm{~m}^{2}=45 \%$ ) et petits (entre 2500 et $5000 \mathrm{~m}^{2}=40 \%$ ).

Tableau 2

Évolution de la gestion du vol à l'étalage par les hypermarchés selon leur taille

\begin{tabular}{|c|c|c|c|c|}
\hline \multicolumn{5}{|c|}{ Nombre annuel moyen d'interpellations } \\
\hline & petits & moyens & gros & ensemble \\
\hline 1989 & 302 & 536 & 827 & 565 \\
\hline 1990 & 310 & 547 & 844 & 574 \\
\hline 1991 & 276 & 517 & 912 & 560 \\
\hline 1992 & 301 & 528 & 972 & 581 \\
\hline 1993 & 284 & 518 & 1091 & 597 \\
\hline \multicolumn{5}{|c|}{ Taux annuel moyen (en \%) d'appels à la police ou à la gendarmerie } \\
\hline & petits & moyens & gros & ensemble \\
\hline 1989 & 20.1 & 20.8 & 23,0 & 21,1 \\
\hline 1990 & 21,8 & 21,2 & 25,6 & 22,4 \\
\hline 1991 & 25,7 & 20,3 & 22,8 & 21,9 \\
\hline 1992 & 28,0 & 23,7 & 23,2 & 24,5 \\
\hline 1993 & 25,4 & 23,3 & 24,3 & 23,9 \\
\hline \multicolumn{5}{|c|}{ Taux annuel moyen (en \%) de formulaires DPS } \\
\hline & petits & moyens & gros & ensemble \\
\hline 1989 & 14,1 & 31.4 & 38.0 & 30,9 \\
\hline 1990 & 22,3 & 32,4 & 40,0 & 33,1 \\
\hline 1991 & 27.0 & 33,3 & 37.9 & 33.4 \\
\hline 1992 & 30,3 & 34.6 & 33,6 & 33.7 \\
\hline 1993 & 25,5 & 34,7 & 33,7 & 32,7 \\
\hline
\end{tabular}


Les gros hypermarchés ont connu en cinq années une progression des interpellations tandis que les petits et moyens connaissaient un tassement, sinon une légère décrue. La hausse constatée dans les gros établissements ne peut être interprétée comme une variation des vols effectivement commis, mais plutôt comme l'indice d'une meilleure efficacité des agents de vigilance dans la dêtection des vols. Les établissements petits et moyens alertent aujourd'hui plus fréquemment qu'hier la police, se hissant désormais à des taux avoisinant ceux des gros établissements (une interpellation sur quatre vols constatés). Ces derniers ont, quant à eux, des pratiques plus stabilisées. Les gros établissements, qui ont appliqué plus rapidement et massivement que les autres la procédure DPS dès son origine (en 1989, la différence des pratiques est encore flagrante entre gros et petits), auraient tendance à s'en dêtourner aujourd'hui.

Il en est de même chez les petits, où l'on constate un accroissement de l'usage de la procédure jusqu'en 1992. On peut se dernander si, avec la chute amorcée en 1993, on n'assisterait pas à une amorce d'alignement sur les gros en vertu d'une loi selon laquelle la découverte, l'expérimentation et le bilan tiré de l'usage d'une procédure censée diminuer un problème massif obéiraient à des cycles d'adaptation et de rejet analogues, avec cependant des rythmes ou temporalités différents. En revanche, l'augmentation régulière de l'utilisation de la procédure DPS attesterait d'un usage entré progressivement dans les mours et habitudes des entreprises de taille moyenne et qui leur conviendrait.

\section{LES MANIFESTATIONS DE LA COOPÉRATION AVEC LES AGENCES PUBLIQUES}

Si la forte corrélation existant entre la taille de l'établissement et le nombre d'interpellations effectuées annuellement est aisément compréhensible, qu'advient-il ensuite des personnes interpellées ? Cherche-t-on à régler, et dans quelle proportion, le sort des voleurs à l'étalage par soi-même, avec la police ou en laissant plutôt le procureur s'en occuper ? Cette question peut être documentée par une réflexion sur le contenu sociologique de la notion de coopération ou de partenariat des victimes collaborant avec les autorités publiques dans le règlement des affaires de vol à l'étalage.

Sachant que les alertes aux polices et les plaintes au Procureur n'obéissent pas nécessairement à des stratégies d'anticipation identiques, on n'est pas surpris de voir apparaître des variations significatives dans les pratiques. De 1989 à 1993, la moyenne des taux d'alerte aux polices publiques (police ou gendarmerie) a oscillé globalement dans une proportion de 21,1 à $24,5 \%$ (autrement dit, pour moins d'un incident sur quatre seulement, les forces de 
police sont informées du flagrant délit de vol à l'étalage par les grandes surfaces). Dans la même période, la moyenne des formulaires de DPS transmis au Parquet a oscillé globalement dans une proportion de 30,9 à 33,7\% (autrement dit, dans presque un tiers des incidents, le Parquet est formellement informé qu'une interpellation en flagrant délit de vol à l'étalage a eu lieu dans les grandes surfaces de son ressort).

Au-delà de ces tendances globales, les pratiques des uns et des autres demeurent fondamentalement différentes. Pour mieux saisir les degrés de coopération avec les services officiels en matière de résolution des contentieux de vols à l'étalage, on a recodé en quatre classes les taux annuels des alertes à la police/gendarmerie et les taux annuels d'utilisation des formulaires de DPS, puis défini par une convention purement statistique en continuum (très peu à très) les services de surveillance des hypermarchés selon leur degré de coopération avec les polices et les services du Procureur (tableau 3).

Tableau 3

Évolution de l'intensité de la coopération des services de vigilance avec polices et Parquet (\%)

\begin{tabular}{|l|c|c|c|c|c|}
\hline $\begin{array}{l}\text { Taux d'appels à la police ou } \\
\text { à la gendarmerie }\end{array}$ & 1989 & 1990 & 1991 & 1992 & 1993 \\
\hline$(*<10 \%):$ très peu coopérants & 39 & 38 & 41 & 33 & 32 \\
$(10 \%<\bullet<30 \%):$ plutôt peu coopérants & 35 & 37 & 37 & 39 & 40 \\
$(30 \%<\bullet<50 \%):$ plutôt coopérants & 12 & 13 & 12 & 17 & 16 \\
$(50 \%<\bullet):$ très coopérants & 14 & 12 & 10 & 11 & 12 \\
\hline Taux de DPS & 1989 & 1990 & 1991 & 1992 & 1993 \\
\hline$(\bullet<10 \%):$ très peu coopérants & 35 & 34 & 33 & 30 & 34 \\
$(10 \%<\bullet<30 \%):$ plutôt peu coopérants & 33 & 32 & 34 & 34 & 31 \\
$(30 \%<\bullet<50 \%):$ plutôt coopérants & 13 & 14 & 14 & 15 & 14 \\
$(50 \%<\bullet):$ très coopérants & 19 & 20 & 19 & 21 & 21 \\
\hline
\end{tabular}

La comparaison sur les cinq années des évolutions conjuguées des fréquences de coopération des services avec les agences publiques conduit à formuler les observations suivantes :

- Les hypermarchés dits très peu coopérants vis-à-vis de la police ou de la gendarmerie ont eu un peu moins tendance à alerter policiers et gendarmes lors d'un flagrant délit de vol à l'étalage au cours des deux dernières années, et à réutiliser un peu plus volontiers la procédure de DPS, comme s'ils jouaient les services du Parquet contre ceux de la police.

- Un phénomène inverse, plus marqué, s'observe dans les établissements dits plutôt peu coopérants, les Procureurs étant moins souvent actionnés que les polices ne sont informées. 
- Dans les hypermarchés dits plutôt coopérants, les alertes à la police ou à la gendarmerie tendent à s'accroître sur l'ensemble de la période étudiée, et à rester stables en ce qui concerne l'usage de la DPS.

- Les hypermarchés dits très coopérants connaissent des pratiques dans l'ensemble plutôt stables; ils auraient plutôt tendance à diminuer leurs pratiques d'alerte aux forces de police, et plutôt tendance à faire un usage plus intensif des formulaires de DPS au Parquet.

Tout se passe donc globalement comme si les marges d'autonomie acquises par les hypermarchés dans la gestion du contentieux des vols à l'étalage avaient tendance à s'essouffler aujourd'hui, après quelques années de pratique et leçons tirées de ces pratiques. Cela est-il dû au fait que les interpellations pour flagrant délit de vol à l'étalage sont devenues de plus en plus délicates à résoudre avec le temps ? C'est-à-dire des objets de conflits plus systématiques que naguère ? Cela est-il dû à un besoin de coopération plus assidu mais différent d'avec les autorités publiques ? Difficile à dire. On peut néanmoins avancer en montrant comment les justifications des pratiques des dirigeants d'hypermarchés (systèmes de rationalités subjectives au sens weberien du terme) informent tendanciellement l'état des rapports entretenus avec les autorités publiques. Il existe d'abord des systèmes de justification attestant que l'on est plus ou moins prédisposé à régler un vol à l'étalage par le biais de son seul système de protection (régulation privée pure), et des systèmes de justification qui montrent qu'on est plutôt prédisposé à le faire prendre en charge par la police ou la gendarmerie (en fonction des attentes instrumentales assignées aux objectifs, pouvoir et légitimité de ces agences publiques).

\section{Dans quelles hypothèses le manager d'un service privé informe-t-il les polices?}

On a proposé une dizaine de cas de figures possibles à partir desquelles des choix en termes d'alerte plutôt systématique ou plutôt rare sont pratiqués suite à l'interpellation d'un auteur appréhendé en flagrant délit de vol à l'étalage.

Les taux de réponse en termes d'alerte plutôt rare sont très importants. Quatre circonstances provoquent des taux importants d'évitement dans les réponses (pas de politique précise). C'est le cas de $30 \%$ de répondants dans l'hypothèse où l'auteur du vol est inconnu du service; il s'agit de ne pas fonder une pratique sur une réputation, un préjugé à l'encontre d'un délinquant primaire. D'autres répondants, pour un taux de $20 \%$, adoptent une position idoine quand l'auteur reconnaît le vol, quand l'auteur restitue ou rembourse la marchandise volée, ou quand il a volé des produits de première 
nécessité, autant d'indicateurs de bonne volonté depuis longtemps analysés par les commerçants dans une catégorie criminologique de sens commun très opératoire, comme celle de vol d'impulsion, vol sans gravité sur le plan de l'intentionnalité ou de la perte. Instrumentalement, on peut penser que les réponses d'indulgence ont pour souci d'éviter une stigmatisation des auteurs par une publicisation disproportionnée de leur acte, avec cette conviction que l'investissement dans la recherche d'une sanction ne sera pas récompensé par le coût de la procédure formellement engagée.

En revanche, trois circonstances conduisent, dans une proportion massive, à demander plutôt systématiquement l'intervention de la police ou de la gendarmerie (proportions de 85 à $95 \%$ ) : elles ont pour caractéristique commune le comportement récalcitrant de l'auteur interpellé qui se rebelle et menace les agents, qui refuse de décliner son identité ou bien nie les faits, autant de preuves qu'il récuse la légitimité de la police privée des commerçants. Pour chacune de ces propositions, une demande d'aide officielle à la police ou à la gendarmerie est la pratique courante, surtout si l'on craint un conflit avec les agents de protection pouvant s'envenimer et provoquer des violences. Au moins s'agit-il de ne pas passer pour tolérant ou faible, ou encore de se montrer déterminé à publiciser l'affaire, et à en espérer des suites conformes à la nécessité de faire un exemple.

La qualité de mineur attachée à la personne du voleur provoque, dans une proportion de $10 \%$, l'appel à d'autres prises en charge (parents ou maîtres).

\section{Comment justifie-t-on le dépôt de plainte simplifié auprès du Procureur?}

En dehors des justifications ritualistes et suivistes arrimées aux rationalités gestionnaires de l'entreprise de distribution (parce que c'est la consigne du groupe commercial auquel on appartient), les principales justifications ont à voir avec les règles de la vie des affaires. et notamment des questions d'économie : gain de temps, simplicité de la procédure. Le fait de donner un semblant de légalité à la procédure permet encore de ne pas perdre la face, lorsqu' une tentative de composition à l'amiable avec le voleur interpellé a échoué, ou n'aurait pas donné les résultats escomptés. D'une façon assez surprenante, d'aucuns avouent d'ailleurs qu'une telle procédure garantirait la légalité d'une transformation à l'amiable du vol de la marchandise en achat ${ }^{4}$.

4. Il semble bien que menacer le voleur des foudres de la justice l'amène parfois à reconnaître ses torts. Il s'agit d'un petit chantage, parfois payant, au moment de l'établissement du dépôt de plainte, pour voleurs influençables. Cette politique n'a toutefois aucune chance 
D'autres opinions sont plutôt liées aux justifications publiques : tantôt on évoque l'efficacité de la mesure (elle est dissuasive parce qu'elle permet au Parquet de lutter contre la récidive; elle dissuade d'autres voleurs potentiels), précisant qu'en deçà d'un taux de préjudice fixé par le Procureur, on n'entreprend rien, anticipant le fait qu'il classera l'affaire. Ce qui n'est évidemment pas l'esprit dans lequel fut pensée la procédure, mais montre néanmoins comment on en a très rapidement anticipé les effets pervers ${ }^{5}$. On entend jouer la carte de la légalité, c'est-à-dire faire montre de bonne volonté à l'égard des autorités judiciaires : on évoque alors la transparence des procédures utilisées mais aussi la défense de l'image de marque de l'enseigne auprès du public. Ce qui démontre, une fois encore, que le sens des affaires peut fort bien s'accommoder d'un certain légalisme. Bref, que l'on croie ou feigne de croire à l'efficacité de cette procédure de DPS, la coopération des hypermarchés avec les autorités policières et judiciaires dans les contentieux de vol à l'étalage, quand elle n'est pas évitée, constitue bel et bien un atout, une ressource, ou un joker, dont il convient d'examiner attentivement la teneur.

\section{DE LA CONFIANCE DANS LES RÉSEAUX INTER-ORGANISATIONNELS}

Ce que l'on pense de l'efficacité de la police ou de la gendarmerie et de la politique pénale conduite par le Parquet conditionne à l'évidence les pratiques de protection mises en ouvre à l'égard des auteurs ou fauteurs de troubles. Dans les registres de la défiance ou de la confiance se redéfinissent la plupart des pratiques de la protection privée. Pour rendre compte des prédispositions des distributeurs à gérer ou non les problèmes par leurs propres moyens, la méthode fut d'agréger les systèmes d'opinions formant des images ou perceptions cohérentes sur l'efficacité du travail des polices publiques, du fonctionnement de la justice pénale, et de la prévention sociojudiciaire. Les attitudes qui en découlent ont des chances d'informer, plus que tout discours formel, sur les pratiques de coopération réelles. À l'aide d'une analyse factorielle des correspondances que le cadre de cet article ne permet pas d'illustrer, on a mis en évidence deux grands systèmes d'oppositions entre les commerçants :

de marcher pour les voleurs qui fanfaronnent, connaissant le tarif du préjudice en deçà duquel aucune poursuite n'aurait lieu. S'il n'y a pas de relais avec le Parquet ou la police capables de démentir sur le champ, le commerçant perd la face dans l'épreuve de force qui s' instaure.

5. C'est oublier aussi que l'on peut toujours déclencher par soi-même l' action publique en tant que victime selon des modalités plus traditionnelles, ne serait-ce que pour faire un exemple et montrer que l'on maîtrise les procédures. 
- Un premier clivage oppose les services les moins coopérants avec les agences pénales à des services très coopérants avec la police et plutôt coopérants avec la justice. Les dirigeants des premiers présentent tous des opinions de style mitigé ou conformiste à l'égard de l'hypothèse de la meilleure efficacité de la gendarmerie sur la police ${ }^{6}$, de la relative impuissance de la police face aux flagrants délits de vol, et de la nécessité de la médiation pénale. Ils s'opposent aux dirigeants ayant des positions moins conformistes qui contestent la prétendue supériorité de la gendarmerie sur la police, le prétendu laxisme des Procureurs classant trop facilement les affaires de vols, et la nécessité de la médiation pénale.

- Un deuxième clivage organise une autre opposition entre des gestionnaires très coopérants avec la police et le Parquet, à des gestionnaires plutôt peu coopérants. Les premiers approuvent plutôt mollement la nécessité de la médiation pénale et de l'admonestation des jeunes, et approuvent fortement l'idée que le Parquet, ayant trop tendance à classer les affaires, n'encourage pas à porter plainte. Les seconds, à l'inverse, manifestent une très vive approbation pour la médiation pénale et l'admonestation des mineurs, contestent l'idée d'un mauvais retour de l'information judiciaire sur le suivi, voire le bien fondé d'une prise en charge policière systématique des interpellés.

En résumé, défiance ou relative confiance à l'égard des agences pénales sont les attitudes qui organisent le mieux l'information disponible sur les pratiques de coopération. Parmi les sensibilités des gestionnaires plutôt enclins à la méfiance à l'égard des autorités publiques, deux sous-groupes de commerçants se distinguent. Le premier est constitué de commerçants critiquant de façon ritualiste l'action de la justice et des polices avec laquelle ils coopèrent peu. Cela s'explique en partie par le fait qu'ils ont en général plutôt peu de problèmes de délinquance à régler, qu'ils ne pratiquent qu'une vigilance diffuse, et qu'étant peu habitués à gérer la protection et la sécurité relevant à leurs yeux de l'État, les affects à ce sujet provoquent en eux une forme d'aveuglcment idéologique. Le deuxiène groupe se distingue par sa relative confiance envers l'action dissuasive des polices qu'il mobilise souvent, mais aussi par sa déception, pour ne pas dire sa défiance à l'égard des juges. Il a tendance à accuser ces derniers de se désintéresser des problèmes de sécurité sur les sites commerciaux. Obligés d'investir dans la surveillance, ces gestionnaires la sous-traitent en faisant appel aux ressources

6. Selon un préjugé assez enraciné dans le corps social français, la gendarmerie (police militaire), censée travailler sans état d'âme, est créditée d'un meilleur professionnalisme et d'une meilleure efficacité judiciaire que la police, corps civil. Pour une vérification empirique de ce postulat, voir Zauberman, 1997. 
du marché des services, mais s'y résolvent difficilement et toujours à contrecour.

Parmi les sensibilités des gestionnaires plutôt enclins à la confiance à l'égard des autorités publiques, encore deux sous-groupes de commerçants se différencient. Les premiers, les moins conformistes, se distinguent par leur confiance envers l'action du Parquet qu'ils jugent positivement, et qu'ils mobilisent du reste souvent par le truchement de la procédure DPS. De même, ils refusent d'adhérer au préjugé de non-efficacité de la police, et la mobilisation de leur service de sécurité plutôt intégré les incite à s'accommoder de la nécessité de partager les tâches : ils essaient d'organiser le plus harmonieusement possible la coopération de leur service avec les polices publiques. Le deuxième groupe est constitué de gestionnaires plutôt confiants envers la justice et convaincus des vertus de l'admonestation policière. Ils se distinguent des précédents par leur plus grande adhésion à toutes solutions alternatives au traitement répressif d'une délinquance de masse comme celle du vol à l'étalage. Dotés de services de vigilance sous-traités, à leurs yeux efficaces, ils se passent plus aisément de l'aide des services répressifs officiels pour régler les comportements problématiques sur leur territoire, au moins pour les contentieux des vols à l'étalage sans violence.

C'est en réalité dans cette configuration que se conçoit et s'expérimente une protection débordant largement les objectifs de la prévention situationnelle. Les préoccupations de prévention sociale des délinquances n'y sont jamais perdues de vue par rapport aux autres.

En la matière, on rencontre certes des partisans du tout ou rien (dans le très court terme), mais également des professionnels aux pratiques beaucoup plus nuancées dans la gestion des risques. Lorsqu'on observe plus qualitativement le sens attribué par cette fraction de managers à des actions de vigilance devant porter des fruits à plus long terme (Ocqueteau, 1993 ; Ocqueteau et Pottier, 1995a), on remarque que l'expérience est d'abord conçue comme un outil de protection contre les manifestations des désordres les plus graves et, une fois les règles du jeu redéfinies, comme un outil quotidien de prévention des actes de petite délinquance troublant l'ambiance. Cette démarche à double détente présente, par rapport aux autres solutions à court terme, d'indéniables chances de succès. Les managers qui l'ont initiée ont conscience que leur entreprise doit jouer son rôle d'inclusion et d'insertion des jeunes en difficulté issus des quartiers de sa zone de chalandise. De fait, outre le métier de vendre, ils participent, à travers des actions citoyennes, à des actions d'animation locale et à des actions de prévention débordant le cadre du périmètre de leur site. Surtout, ils s'engagent à fournir du travail aux jeunes en difficulté, achevant par là le processus d'intégration ouverte sur la cité, plutôt que de devenir des bulles de sécurité complètement repliées sur elles-mêmes 
et peu intéressées aux actions sur les causes sociales de l'insécurité, à l'extérieur.

\section{CONCLUSION : REMARQUES SUR LA PRÉVENTION LOCALE DE L'INSÉCURITÉ URBAINE}

La protection des espaces privés ouverts au public par la mise au point de systèmes de vigilance dans les organisations administratives ou commerciales s'est empiriquement bricolée en France dans les années 1980, au gré d'une perception renouvelée des risques et des vulnérabilités de leurs dirigeants. La prévention des risques ne concerne en effet plus seulement l'accident du travail ou l'incendie, elle met désormais sur le même plan de la gestion des risques certaines formes de délinquance urbaine qui se développent dans ces espaces. Si la vigilance privée s'y est longtemps exercée dans l'infra-droit, il ne fait pas de doute que, sociologiquement, elle constitue une authentique police de l'organisation jouant par définition un rôle de police de proximité sur un territoire restreint. Elle est admise comme telle par la plupart des visiteurs des espaces commerciaux. Cette évolution sociologique est d'ailleurs entérinée par les pouvoirs publics français qui, pour la première fois, en 1995, se sont officiellement convertis à la nécessité de réorganiser le système policier français en tenant compte de l'existence et de l'apport de l'ensemble des agents, y compris privés, concourant à la production de la sécurité collective (Ocqueteau, 1997).

Il convient en effet de préciser que, durant toute la décennie 1980, la politique pénale instituée par le gouvernement français converti au modèle de prévention sociale de la délinquance avait cherché à mieux impliquer les collectivités locales ou municipalités dans cette politique, sans particulièrement cibler l'action en direction des organisations les plus vulnérables à la petite délinquance. Fait significatif, il a fallu attendre 1993 pour qu'un comité interministériel, le Conseil national des Villes, réoriente sa réflexion sur la police de prévention des désordres urbains, envisageant pour la première fois une doctrine d'action pour des polices dites de la Ville : co-produire de la sécurité collective à côté des policiers d'État et des policiers municipaux. Le document de base (Conseil national des villes, 1993) visait les régisseurs du logement social dans les cités d'habitation à loyers modérés, les conducteurs et receveurs dans les transports en commun, les vigiles et agents de surveillance dans les centres commerciaux, sans se soucier outre mesure d'articuler le statut des agents visés à la nature de l'espace où ils se déploient. $\mathrm{Ce}$ document focalisait simplement son diagnostic sur des lieux particulièrement sensibles : l'école, le logement social, les transports publics, les centres commerciaux. Il recommandait de faire reculer le sentiment d'insécurité expliqué notamment par les vols à l'étalage ou à l'arraché, la délinquance 
sur les parkings, la présence de marginaux, les problèmes liés à la drogue susceptibles de dégénérer en incidents graves.

Le ministère de l'Intérieur, à travers une loi cadre de 1995, entérina certains aspects de cette nouvelle philosophie d'action sans pour autant afficher d'appels à la communauté et au partenariat local dans des stratégies de responsabilisation du public, comme c'est fréquemment le cas outre Manche et dans le monde anglo-saxon (Crawford, 1997). Il n'en appela pas non plus à un usage intensif des ressources du marché de la protection. Prévention sociale des délinquances, médiations pénales et justice de proximité constituent certes des programmes valorisés, mais il ne s'agit pas à proprement parler de community policing. Îlotage public ou police de proximité publique sont les seules acceptions admises en pratique. Les raisons en sont connues : en France, la notion de communauté heurte l'idée du pacte républicain (Wieviorka, 1996) basé sur l'universalité de la citoyenneté. Et cela, en dépit d'autres réalités attestant de la montée de différences culturelles, de différences de localisations urbaines, voire de différences ethniques parmi les populations. Dans les discours publics, le thème de l'inclusion sociale reste toujours valorisé, même si, dans des domaines très variés, l'on observe des pratiques d'exclusion de plus en plus fréquentes (Paugam, 1996), y compris dans certains espaces commerciaux où l'interdiction de paraître faite à certaines populations constitue souvent une pratique officieuse de leurs dirigeants. En matière de justice, les procureurs contrôlent encore les différentes formules de justice de proximité et de médiation pénale (Faget, 1997 ; Wyvekens, 1997). Enfin, la faiblesse historique du mouvement associatif de prise en charge des déviances (Robert, 1997) liée au processus de centralisation historique de la fonction publique a produit une police particulièrement jalouse de son monopole dans le domaine de la sécurité des personnes et des biens. Elle demande en conséquence à être associée à toute réforme qui tendrait à revaloriser le rôle des autres acteurs publics (policiers municipaux) ou non publics du policing, sous peine de la faire capoter. De ce point de vue, Monjardet (1996) a fort bien expliqué les raisons des blocages historiques, culturels-identitaires et organisationnels de la police française dans le domaine de la gestion de la délinquance urbaine au quotidien. Ils devront être dépassés par un important effort d'adaptation de la police publique à ces nouveaux défis, sous peine que celle-ci se voit durablement discréditée auprès des citoyens en demande d'un surcroît de sécurité.

Voilà autant de paramètres décisifs qui expliquent les raisons pour lesquelles toute évaluation criminologique du travail de proximité des agents de gardiennage ou de protection, dans quelque organisation ouverte que ce soit, ne peut être conduite en France qu'en connexion avec les anticipations de leurs managers vis-à-vis de l'efficacité du travail des polices publiques 
(centrales ou locales). Nous croyons l'avoir démontré empiriquement dans la présente étude. Sauf à devoir en systématiser le genre dans d'autres domaines et sous réserve d'infirmations, celle-ci nous paraît rendre largement caducs les débats criminologiques théoriques (encore trop fortement mâtinés d'idéologie) sur les mérites respectifs des modèles de prévention sociale ou de prévention situationnelle. En dehors du thème de la prévention des communautés contre le crime qui n'a aucun ancrage réel en France, le thème de la prévention policière du crime en partenariat avec les nouveaux agents de la sécurité (bien souvent des habits neufs sous des fonctions anciennes (concierges)) ne laisse guère le choix à ces derniers que de se couler dans d'autres rôles que ceux de la répression, de l'éducation, ou du travail social. En France du moins, il semble que le mélange des genres soit un exercice difficile et de portée limitée. Dans les fonctions de policing, l'identité professionnelle de l'ensemble des agents concernés s'évalue toujours à l'aune de celle des policiers publics.

\section{RÉFERENCES}

AUBUSSON, B. (1997), «Les statistiques policières : que compte-t-on et comment ? ", Questions pénales, vol. $\mathrm{X}, \mathrm{n}^{\circ} 3$.

BAYLEY, D. H. et SHEARING, C. D. (1996), « The future of policing ", Law and Society Review, vol. 30, $\mathrm{n}^{\circ} 3$, p. 585-606.

BECK, A. et WILLIS, A. (1995), Crime and Security ; Managing the Risk to Safe Shopping, Leicester, Perperuity Press.

CONSEIL NATIONAL DES VILLES (1993), Les polices dans la ville, Paris, C.N.V.-D.S.U.

CRAWFORD, A. (1997), The Local Governance of Crime, Appeals to Community and Partnerships, Oxford, Clarendon Press.

DAVIS, M. G., LUNDMAN, R. J. et MARTINEZ, R. Jr (1991), « Private corporate justice : store police, shoplifters, and civil recovery 》, Social Problems, vol. 38, n' 3, p. 395 411.

FAGET, J. (1997), La médiation, essai de politique pénale, Toulouse, Erès.

FARRINGTON, D. P., BOWEN, S., BUCKLE, A., BURNS-HOWELL, T., BURROWS, J. et SPEED, M. (1993), «An experiment on the prevention of shoplifting », in CLARKE R.V. (dir.), Crime Prevention Studies, (p. 93-119), Criminal Justice Press, Monsey-New York.

FARRINGTON, D. P. et BURROWS, J. N. (1993), « Did shoplifting really decrease ? », British Journal of Criminology, vol. 33, $\mathrm{n}^{\circ} 1$, p. 57-69.

FAUGERON, C., FICHELET, M. et ROBERT, Ph. (1977), Le renvoi du déviant : des modes informels aux systèmes institutionnels de contrôle de la déviance, Paris, CORDES.

FEUERVERGER, A. et SHEARING, C. D. (1982), "An analysis of the prosecution of shoplifters ", Criminology, vol. XX, nº 2, p. 273-289.

HIBBERT, M. et SHAPLAND, J. (1993), Violent Crime in Small Shops, London, The police fundation.

KERCKVOORDE J. van, (1978), « Le ministère public et la poursuite du vol à l'étalage, une étude empirique ", Déviance et société, vol. $2, \mathrm{n}^{\circ} 4, \mathrm{p}$. 365-383. 
MONJARDET, D. (1996), Ce que fait la police, sociologie de la force publique, Paris, La Découverte.

OCQUETEAU, F. (1993), « Les centres commerciaux, cibles d'incivilités et promoteurs de sécurité », Déviance et société, vol. 17, $\mathrm{n}^{\circ} 3$, p. 235-260.

OCQUETEAU, F. (1997), Les défis de la sécurité privée, surveillance et protection dans la France d'aujourd'hui, Paris, L'Harmattan.

OCQUETEAU, F. et HEILMANN, E. (1997), « Droit et usages des nouvelles technologies : les enjeux d'une réglementation de la vidéosurveillance », Droit et société, vol. 3637, p. 331-344.

OCQUETEAU, F. et POTTIER, M.-L. (1995a), «Vidéosurveillance et gestion de l'insécurité dans un centre commercial : les leçons de l'observation », Les Cahiers de la sécurité intérieure. vol. 21 , p. 60-74.

OCQUETEAU F. et POTTIER, M.-L. (1995b), Vigilance et sécurité dans les grandes surfaces, Paris, IHESI-L'Harmattan.

PAUGAM, S. (dir.) (1996), L'exclusion, l'état des sawoirs, Paris, La Découverte.

ROBERT, Ph. (1997), «Évolutions historiques et enjeux actuels des frontières entre public et privé dans le domaine de la sécurité », Séminaire Ville et Hospitalité, Paris, MSHPlan contruction et architecture, p. 153-172, ronéo.

ROBERT, Ph., AUBUSSON de CAVARLAY, B., POTTIER, M.-L. et TOURNIER, P. (1994), Les comptes du crime, les délinquances en France et leurs mesures, Paris, L'Harmattan.

SHEARING, C. D. et STENNING, P. C. (1983), « Private security, implications for social control ", Social problems, vol. 30, n" 5, p. 493-506.

SHEARING, C. D. et STENNING, P. C. (dir) (1987), Private policing, California, Sage.

SIMMAT-DURANT, L. (1994), Orientation et sélection des affaires pénales : une approche quantitative de l'action du Parquet, Paris, Université de Paris I, thèse de doctorat en démographie, inédite.

WIEVIORKA, M. (dir) (1996), Une société fragmentëe? Le multiculturalisme en débat, Paris, La Découverte.

WYVEKENS, A. (1997), L'insertion locale de la justice pénale, aux origines de la justice de proximité, Paris, l'Harmattan.

ZAUBERMAN, R. (1982), « Renvoyants et renvoyés », Déviance et société, vol. 6, $\mathrm{n}^{\circ} 1$, p. 23-52.

ZAUBERMAN, R. (1997), "Le traitement des vols et cambriolages par la gendarmerie nationale », Questions pénales, vol. $\mathrm{X}, \mathrm{n}^{\circ} 2$. 